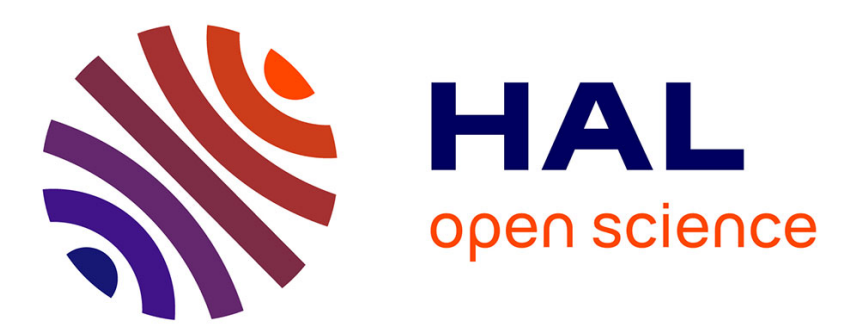

\title{
Ultrasonic fragmentation of microbubbles: a theoretical approach of the flash in flash-echo
}

Michiel Postema, Georg Schmitz

\section{To cite this version:}

Michiel Postema, Georg Schmitz. Ultrasonic fragmentation of microbubbles: a theoretical approach of the flash in flash-echo. 27th Annual International Conference of the IEEE Engineering in Medicine and Biology Society, 2005, Shangai, China. pp.4023-4026, 10.1109/IEMBS.2005.1615344 . hal-03195214

\section{HAL Id: hal-03195214 https://hal.science/hal-03195214}

Submitted on 11 Apr 2021

HAL is a multi-disciplinary open access archive for the deposit and dissemination of scientific research documents, whether they are published or not. The documents may come from teaching and research institutions in France or abroad, or from public or private research centers.
L'archive ouverte pluridisciplinaire HAL, est destinée au dépôt et à la diffusion de documents scientifiques de niveau recherche, publiés ou non, émanant des établissements d'enseignement et de recherche français ou étrangers, des laboratoires publics ou privés. 


\title{
Ultrasonic fragmentation of microbubbles: a theoretical approach of the flash in flash-echo
}

\author{
Michiel Postema, Georg Schmitz
}

Institute for Medical Engineering, Ruhr-Universität Bochum, Bochum, Germany

\begin{abstract}
Predicting the dynamic behavior of ultrasound insonified lipid-shelled microbubbles has been of much clinical interest. For perfusion measurements, a technique named flashecho has been proposed. A burst of high-MI ultrasound is to destroy the contrast agent bubbles, supposedly resulting in a strong scattering signal that is visible on the B-mode image: the flash. The absence of this strong response in parts of the B-mode image indicates a (too) low perfusion. In this paper, we investigate how microbubbles collapse and fragment. An overview of fragmentation theory is given, followed by some high-speed optical observations of collapsing and fragmenting microbubbles in an ultrasonic field. Fragmentation occurs exclusively during the collapse phase. We hypothesize that fragmentation will only occur if and only if the kinetic energy of the collapsing microbubble is greater than the instantaneous bubble surface energy. In contradiction to the assumption that the Blake critical radius is a good approximation for a fragmentation threshold, our simulations show $R_{\max } / R_{0} \ll 2$ for most microbubbles.
\end{abstract}

\section{INTRODUCTION}

Ultrasound contrast agents consist of gas bubbles with equilibrium radii $R_{0}$ up to $5 \mu \mathrm{m}$. These microbubbles are small enough to be transported intravascularly and to pass through capillary vessels. Because their resonance frequencies

$$
f_{\mathrm{r}}[\mathrm{MHz}] \approx \frac{3.3\left[\mu \mathrm{m} \mu \mathrm{s}^{-1}\right]}{R_{0}[\mu \mathrm{m}]},
$$

coincide with those applied in ultrasonic imaging, they are suitable markers for the detection of perfused areas [1]. The only ultrasound contrast agent currently allowed for clinical use in Europe is SonoVue ${ }^{\mathrm{TM}}$. It is a second generation ultrasound contrast agent, consisting of $\mathrm{SF}_{6}$ gas microbubbles encapsulated by an elastic lipid shell. Predicting the dynamic behavior of ultrasound insonified lipid-shelled microbubbles has been of much clinical interest. For perfusion measurements, a technique named flash-echo has been proposed. A burst of high-MI ultrasound is to destroy the contrast agent bubbles, supposedly resulting in a strong scattering signal that is visible on the B-mode image: the flash. The absence of this strong response in parts of the B-mode image indicates a (too) low perfusion [2].

To enhance flash-echo techniques, the ultrasonic destruction of contrast agent microbubbles has to be understood. Previously, we presented a quantitative overview of contrast agent microbubble destruction mechanisms, based on highspeed optical observations [3]. We found that fragmentation is the most occurring destruction mechanism for microbub- bles with an elastic shell. Fragmentation is the fission of a microbubble into a number of smaller microbubbles.

In this paper, we investigate microbubble collapse and fragmentation. An overview of fragmentation theory is given. We also present high-speed optical observations of fragmenting microbubbles in an ultrasonic field.

\section{THEORY}

The shell of SonoVue ${ }^{\mathrm{TM}}$ consists of a lipid monolayer that, under the conditions of our experiments, is in a solid state. It behaves like an elastic membrane that ruptures under relatively small strain [4]. At relatively high acoustic amplitudes (mechanical index $\mathrm{MI} \approx 0.6)^{1}$, lipid-encapsulated microbubbles may expand to more than 10-fold their initial surface areas before coalescing [5]. By the time of maximal expansion, therefore, the shell has ruptured, leaving newly formed clean free interfaces [5]. As such, the elastic properties of the shell will have diminished, resulting in maximal expansions similar to free gas bubbles [6]. Therefore, we neglect the influence of the shell in this section.

In this section, we describe the growth, oscillation, and fragmentation of a microbubble in an infinite liquid subjected to an ultrasonic field. If the insonifying frequency $f \ll f_{\mathrm{r}}$, the pressure in the liquid $p_{\mathrm{L}}$ changes very slowly and uniformly compared to the natural time scale of the microbubble [7], [8]. The radius of a bubble $R$ in response to quasistatic changes in the liquid pressure is described by [8]:

$$
p_{\mathrm{L}}=\left(p_{0}^{\infty}-p_{\mathrm{v}}+\frac{2 \sigma}{R_{0}}\right)\left(\frac{R_{0}}{R}\right)^{3 \gamma}+p_{\mathrm{v}}-\frac{2 \sigma}{R} .
$$

Here, $p_{0}^{\infty}$ is the static pressure of the liquid, $p_{\mathrm{v}}$ is the vapor pressure, $R_{0}$ is the equilibrium radius of the microbubble, $\gamma$ is the polytropic exponent, and $\sigma$ is the surface tension. Figure 1 shows the right-hand-side of eq. (2), for different $R_{0}$. Each curve has a minimum in $\left(R_{\mathrm{c}}, p_{\mathrm{L}}\right)$, where $R_{\mathrm{c}}$ is the critical Blake radius and $p_{\mathrm{L}_{\mathrm{c}}}$ is the critical liquid pressure. The region to the right of the Blake radius represents unstable equilibrium conditions [7], [9]. If the liquid pressure is lowered until it reaches a value below $p_{\mathrm{L}_{\mathrm{c}}}$, no equilibrium radius exists [8], resulting in explosive growth of the bubble, much larger than $R_{0}$. The ambient pressure eventually increases again, during the ultrasonic compression phase, causing the

\footnotetext{
${ }^{1}$ The mechanical index is defined by $\mathrm{MI}=p_{\mathrm{ac}}^{-} / f$, where $p_{\text {ac }}^{-}$is the peak rarefactional acoustic pressure in $\mathrm{MPa}$, and $f$ is the center insonifying frequency in $\mathrm{MHz}$.
} 


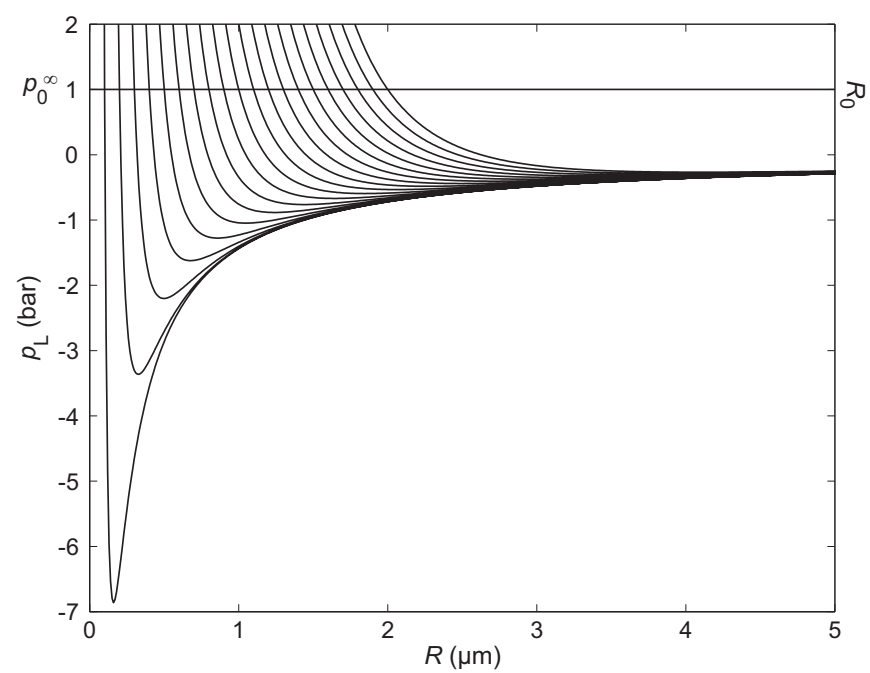

Fig. 1. Solutions of eq. (2) for different equilibrium radii $0.1 \leq R_{0} \leq$ $2.0 \mu \mathrm{m}$, taking $p_{0}^{\infty}=1 \mathrm{~atm}, \gamma=1.4$, and $\sigma=0.072 \mathrm{~kg} \mathrm{~s}^{-2}$.

bubble to collapse violently [10]. The Blake radius has been approximated by

$$
R_{\mathrm{c}} \approx 2 R_{0}
$$

During the initial part of the collapse the acceleration, $\ddot{R}$ is negative. This sign changes as the gas inside the bubble begins to be compressed, and the rebound begins [10]. Microbubble fragmentation has been expected and observed close to this moment, when $\ddot{R}=0$ [3], [11].

For an ideal gas bubble, the oscillating behavior has been described by the so-called RPNNP equation, named after its developers Rayleigh, Plesset, Noltingk, Neppiras, and Poritsky [12]:

$$
\begin{aligned}
& \rho R \ddot{R}+\frac{3}{2} \rho \dot{R}^{2}=p_{\mathrm{g} 0}\left(\frac{R_{0}}{R}\right)^{3 \gamma}+p_{\mathrm{v}}-p_{0}^{\infty} \\
& -\frac{2 \sigma}{R}-\delta \omega \rho R \dot{R}-p_{\mathrm{ac}}(t),
\end{aligned}
$$

where $p_{\mathrm{g} 0}$ is the initial gas pressure inside the bubble $p_{\mathrm{g} 0}=$ $p_{0}^{\infty}-p_{\mathrm{v}}+\frac{2 \sigma}{R_{0}}, p_{\text {ac }}(t)$ is the acoustic pressure in time, $\delta$ is the total damping coefficient, $\rho$ is the liquid density, and $\omega$ is the angular driving frequency $\omega=2 \pi f$. The total damping coefficient is a summation of the damping coefficient due to radiation $\delta_{\mathrm{r}}$, the damping coefficient due to heat conduction $\delta_{\mathrm{t}}$, and the damping coefficient due to the viscosity of the surrounding liquid $\delta_{\mathrm{v}}$ [12], [13]:

$$
\begin{aligned}
\delta & =\delta_{\mathrm{r}}+\delta_{\mathrm{t}}+\delta_{\mathrm{v}} \\
\delta_{\mathrm{r}} & =k R \\
\delta_{\mathrm{t}} & \approx \frac{3}{5}(\gamma-1) \\
\delta_{\mathrm{v}} & =\frac{4 \eta}{\rho \omega R^{2}},
\end{aligned}
$$

where $\eta$ is the viscosity of the liquid, and $k$ is the wave number

$$
k=\frac{\omega}{c},
$$

where $c$ is the speed of sound in the liquid.
For $R_{0} \ll \lambda$, where $\lambda$ is the wavelength of an ultrasonic cycle, $\lambda=2 \pi / k$, the pressure generated by a pulsating microbubble, observed in the far field at distance $r$, can be approximated by [13]:

$$
p \approx \frac{\rho R}{r}(\ddot{R} R+2 \dot{R}) .
$$

The number of fragments into which a microbubble breaks up, is related to the dominant spherical harmonic oscillation mode [10], [6]. For any positive

$$
\Gamma=\frac{\rho R^{2} \ddot{R}}{\sigma},
$$

there must be a mode $n$ for which the spherical harmonic distortion has a maximum. This mode depends on $\Gamma$ by:

$$
n=\frac{1}{3} \sqrt{7+3 \Gamma}-\frac{2}{3} \text {. }
$$

Estimating an approximate fragment radius of [10]:

$$
R_{\mathrm{f}} \approx \frac{R}{n},
$$

the number of fragments $N$ may be taken:

$$
N \approx n^{3} \text {. }
$$

Mode 2 oscillations have been observed with lipidencapsulated microbubbles, leading to fragmentation into 8 newly formed microbubbles [6].

The kinetic energy of a single microbubble in an infinite fluid is given by [10]:

$$
E_{\mathrm{k}}=2 \pi \rho R^{3} \dot{R}^{2} .
$$

The resulting microbubble fragments contain more surface free energy $\sum_{i} E_{\mathrm{f}, i}$ than the single bubble prior to fragmentation $E_{\mathrm{s}}$ :

$$
\sum_{i=1}^{N} E_{\mathrm{f}, i} \approx \frac{4}{3} \pi R_{\mathrm{f}}^{2} \sigma N \approx \frac{4}{3} \pi R^{2} \sigma N^{\frac{1}{3}}=N^{\frac{1}{3}} E_{\mathrm{s}}
$$

We hypothesize that fragmentation will only occur if and only if:

$$
E_{\mathrm{k}}>\left(\sum_{i=1}^{N} E_{\mathrm{f}, i}-E_{\mathrm{s}}\right)
$$

\section{MATERIALS AND METHODS}

\section{A. Simulations}

We simulated the oscillating behavior of free microbubbles with various sizes in a harmonic acoustic field:

$$
p_{\mathrm{ac}}(t)=p_{\mathrm{ac}}^{-} \sin \omega t .
$$

Equations (4)-(13) were computed with MATLAB ${ }^{\circledR}$ (The MathWorks, Inc., Natick, MA) programs. The following fixed parameters were used: $c=1480 \mathrm{~ms}^{-1}, p_{0}^{\infty}=1 \mathrm{~atm}$, $p_{\mathrm{v}}=2.33 \mathrm{~Pa}, \gamma=1.4, \eta=0.001 \mathrm{~Pa} \mathrm{~s}, \rho=998 \mathrm{~kg} \mathrm{~m}^{3}$, and $\sigma=0.072$ $\mathrm{kg} \mathrm{s}^{-2}$. We did not approximate $\delta(R(t))$ by an equilibrium damping coefficient. The acoustic amplitudes modeled correspond to $\mathrm{MI} \ll 2$ (well within the clinical diagnostic range). 


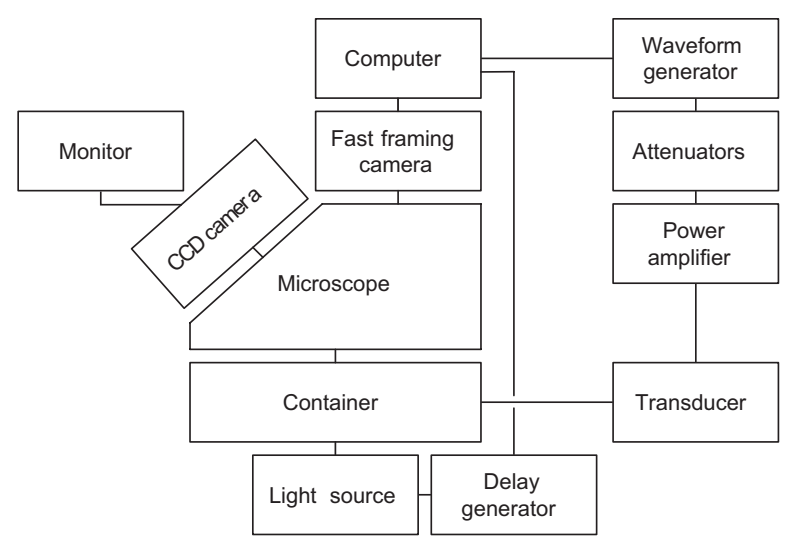

Fig. 2. Schematic overview of the experimental setup.

Here, we focus on simulated driving frequencies of 0.5 $\mathrm{MHz}$ and $2.0 \mathrm{MHz}$, because our optical interrogation was undertaken using these ultrasonic frequencies.

For microbubble radii $0.2<R_{0}<12.0 \mu \mathrm{m}$, the critical acoustic pressures $p_{\mathrm{c}}$ were computed, above which eq. (14) holds. For comparison with the Blake critical radius, the maximal microbubble radii $R_{\max }\left(R_{0}, p_{\mathrm{c}}\right)$ were computed using the RPNNP-equation, and divided by the initial radii $R_{0}$.

\section{B. Optical observations}

For the observations of lipid-encapsulated microbubble fragmentation, we made use of high-speed photographs. The data had been captured at the Department of Experimental Echocardiography, Thoraxcentre, Erasmus MC, Rotterdam, The Netherlands, with use of an Imacon 468 fast framing camera system (DRS Hadland, Ltd., Tring, UK), taking eight two-dimensional frames at $3 \mathrm{MHz}$. In all observations, the first frame was taken a few microseconds before ultrasonic waves reached the contrast agent. The other seven frames were taken during ultrasound insonification, with $0.33 \mu \mathrm{s}$ interframe time for $500-\mathrm{kHz}$ ultrasound, spanning a full ultrasonic cycle. An overview of this experimental setup is shown in Fig. 2. It has been more extensively described in Ref. [14].

We investigated an experimental ultrasound contrast agent (Bracco Research SA, Genève, Switzerland). It consists of phospholipid-encapsulated gas bubbles ranging in diameter from 1 to $6 \mu \mathrm{m}$ with a median of $2 \mu \mathrm{m}$. Its acoustic behavior resembles that of SonoVue ${ }^{\mathrm{TM}}$ [15]. Undiluted ultrasound contrast agent $(5 \mathrm{ml}$ of a $0.9 \%$ sodium chloride dilution, added to a $25 \mathrm{mg}$ vial) was inserted through a cellulose Cuprophan ${ }^{\circledR}$ capillary (Membrana GmbH, Wuppertal, Germany) with a $200 \mu \mathrm{m}$ diameter. The capillary was fixed in the focal area of an ultrasonic transducer.

\section{RESUlts AND Discussion}

Examples of high-speed photographs microbubble fragmentation are presented in Fig. 3. Fragmentation occurs during collapse. In most observations of fragmenting contrast agent microbubbles, other bubbles can be observed
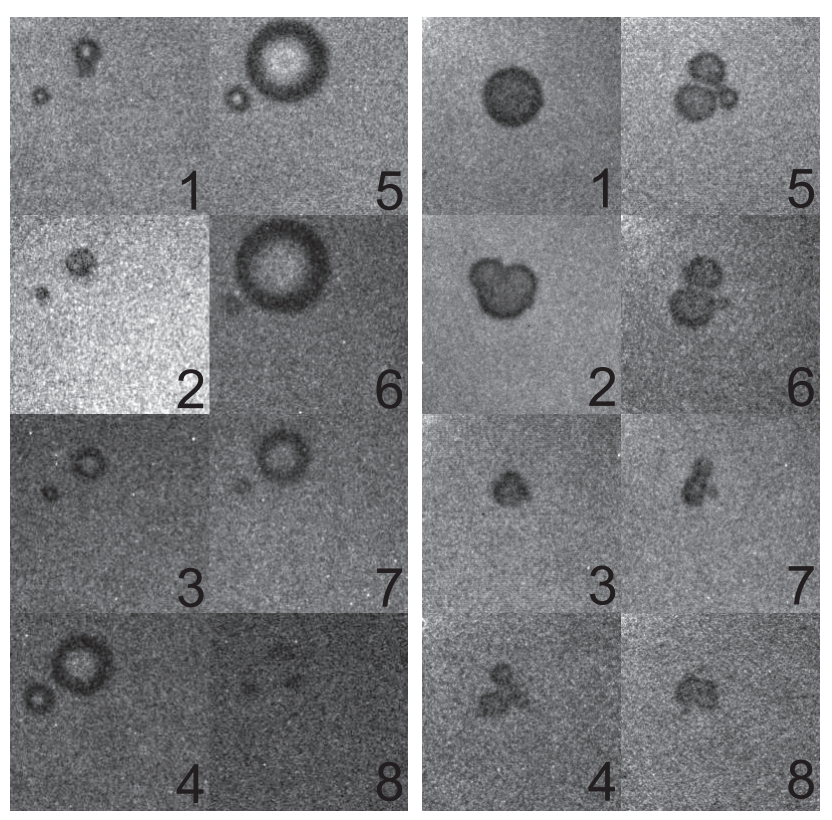

Fig. 3. Two optical image sequences showing fragmentation of lipid-encapsulated contrast agent microbubbles during insonification at $f=0.5 \mathrm{MHz}$ and $\mathrm{MI}<1$. The frames were captured at 3 million frames per second. Frames 1 have been taken prior to ultrasound arrival. The other seven frames in a sequence cover one full ultrasonic cycle. Each frame corresponds to a $30 \times 30 \mu \mathrm{m}^{2}$ area. Left: $R_{0}=1.5 \mu \mathrm{m}, R_{\max }=7 \mu \mathrm{m}, N>7$. An $R_{0}=1 \mu \mathrm{m}$ microbubble is present to the lower left at $r=10 \mu \mathrm{m}$ distance. Right: $R_{0}=4.5 \mu \mathrm{m}, N>3$. The images are courtesy of M. Postema and N. de Jong, Department of Experimental Echocardiography, Thoraxcentre, Erasmus MC, Rotterdam, The Netherlands.

close to the fragmenting bubble. If nearby microbubbles are close enough to an oscillating microbubble, these may induce surface instabilities that have been associated with fragmentation. After fragmentation, microbubble fragments have been observed to coalesce (merge), reducing the surface energy. An image sequence of the coalescence of free (not
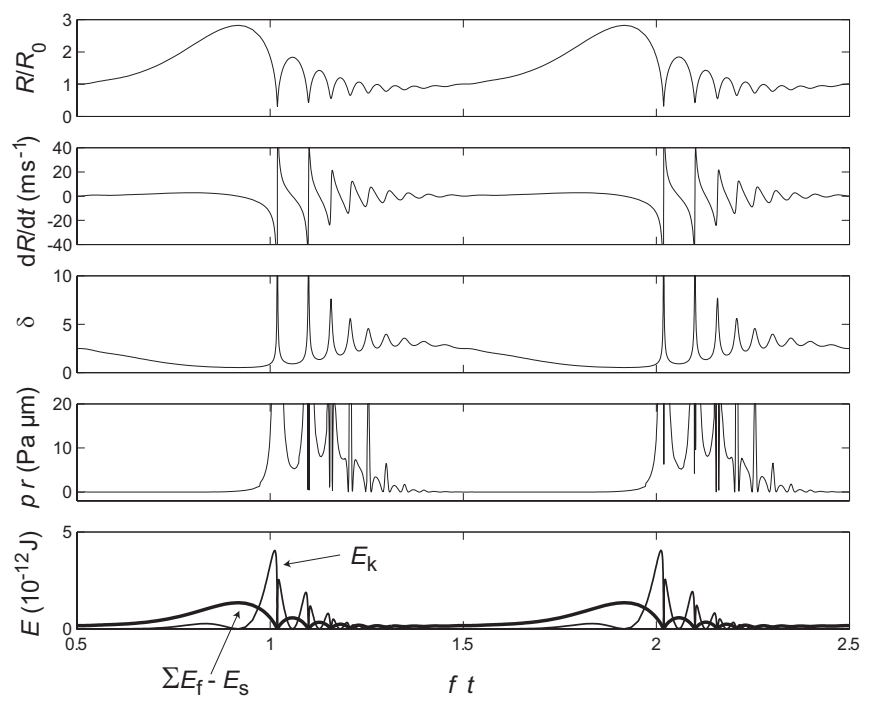

Fig. 4. Simulation of the oscillation behavior of an $R_{0}=0.75 \mu \mathrm{m} \mathrm{mi}-$ crobubble, during insonification at $f=0.5 \mathrm{MHz}$ and $p_{\mathrm{ac}}^{-}=210 \mathrm{kPa} . \sum_{i} E_{\mathrm{f}, i}$ was computed for $N=8$. 


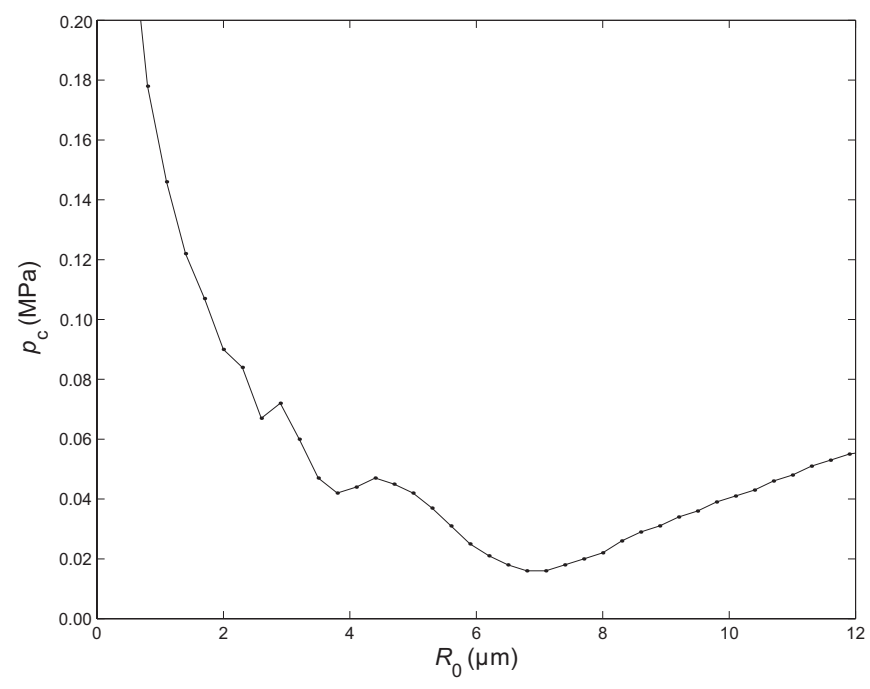

Fig. 5. Critical pressure as a function of initial radius.

encapsulated) microbubble fragments has been presented in [16].

An example of simulated oscillating behavior of a microbubble is shown in Fig. 4. The relatively slow expansion is followed by a rapid collapse. The collapse generates a high acoustic pressure $p$ at distance $r$. This way, the collapsing microbubbles account for the flash. Close to collapse, the kinetic energy of the microbubble becomes higher than the surface energy, as hypothesized. This is the oscillation phase where microbubble break-up has been observed.

Figure 5 shows the critical pressure above which eq. (14) holds. Clearly, the critical pressure is minimal around resonance size $\left(R_{0} \approx 6.5 \mu \mathrm{m}\right)$. Furthermore, local minima can be appreciated at harmonic resonance sizes. At relatively low acoustic pressures, only microbubbles with sizes close to resonance will fragment.

The maximal expansion radii at the critical pressures normalized by the initial radii are demonstrated in Fig. 6. In contradiction to the assumption that the Blake critical radius is a good approximation for a fragmentation threshold, our simulations show $R_{\max } / R_{0} \ll 2$ for most microbubbles.

\section{COnClusions}

Fragmentation occurs exclusively during the collapse phase. We hypothesize that fragmentation will only occur if and only if the kinetic energy of the collapsing microbubble is greater than the instantaneous bubble surface energy. From our simulations it follows that the Blake critical radius is not a good approximation for a fragmentation threshold.

\section{REFERENCES}

[1] P. N. Burns, P. Hilpert, and B. B. Goldberg, "Intravenous contrast agent for ultrasound Doppler: In vivo measurement of small tumor vessel dose-response," Proc. Annu. Int. Conf. IEEE Eng. Med. Biol. Soc., vol. 12, no. 1, pp. 322-324, 1990.

[2] K. Wei, A. R. Jayaweera, S. Firoozan, A. Linka, D. M. Skyba, and S. Kaul, "Quantification of myocardial blood flow with ultrasoundinduced destruction of microbubbles administered as a constant venous infusion," Circulation, vol. 97, pp. 473-483, 1998.

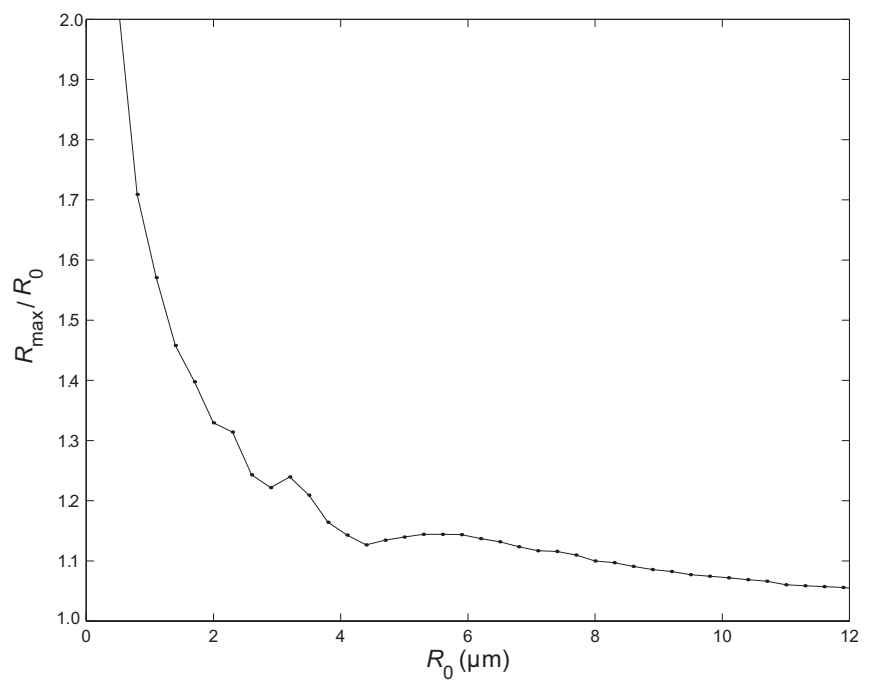

Fig. 6. Relative microbubble excursion as a function of initial radius.

[3] M. Postema, A. Bouakaz, C. T. Chin, and N. de Jong, "Optical observations of ultrasound contrast agent destruction," Acta Acust. united Acust., vol. 89, p. 728, 2003.

[4] Z. Zhou and B. Joós, "Mechanisms of membrane rupture: from cracks to pores," Phys. Rev. B, vol. 56, no. 6, pp. 2997-3009, 1997.

[5] M. Postema, P. Marmottant, C. T. Lancée, S. Hilgenfeldt, and N. de Jong, "Ultrasound-induced microbubble coalescence," Ultrasound Med. Biol., vol. 30, no. 10, pp. 1337-1344, 2004.

[6] M. Postema, A. van Wamel, C. T. Lancée, and N. de Jong, "Ultrasound-induced encapsulated microbubble phenomena," Ultrasound Med. Biol., vol. 30, no. 6, pp. 827-840, 2004.

[7] C. E. Brennen, Cavitation and bubble dynamics. New York: Oxford University Press, Inc, 1995.

[8] A. Harkin, A. Nadim, and T. J. Kaper, "On acoustic cavitation of slightly subcritical bubbles," Phys. Fluids, vol. 11, no. 2, pp. 274287, 1999.

[9] D. Or and M. Tuller, "Reply to comment by N. Kartal Toker, John T. Germaine, and Patricia J. Culligan on "Cavitation during desaturation of porous media under tension"," Water Resour. Res., vol. 35, no. 7, pp. 1949-1964, 1999.

[10] C. E. Brennen, "Fission of collapsing cavitation bubbles," Proc. CAV 2001 4th Int. Symp. Cavitation, 2001.

[11] J. E. Chomas, P. Dayton, D. May, and K. Ferrara, "Threshold of fragmentation for ultrasonic contrast," J. Biomed. Opt., vol. 6, no. 2, pp. 141-150, 2001.

[12] N. de Jong, Acoustic properties of ultrasound contrast agents. Rotterdam: N de Jong, 1993.

[13] A. Ghiotto and J. D. Penrose, "Investigating the acoustic properties of the underwater implosions of light globes and evacuated spheres," Proc. Austr. Acoust. Soc. Conf., 2000.

[14] M. Postema, A. Bouakaz, C. T. Chin, and N. de Jong, "Simulations and measurements of optical images of insonified ultrasound contrast microbubbles," IEEE Trans. Ultrason., Ferroelect., Freq. Contr., vol. 50, no. 5, pp. 523-536, 2003.

[15] J. M. Gorce, M. Arditi, and M. Schneider, "Influence of bubble size distribution on the echogenicity of ultrasound contrast agents: A study of SonoVue ${ }^{\mathrm{TM}}$," Invest. Radiol., vol. 35, no. 11, pp. 661-671, 2000.

[16] M. Postema, P. Marmottant, C. T. Lancée, M. Versluis, S. Hilgenfeldt, and N. de Jong, "Ultrasound-induced coalescence of free gas microbubbles," Proc. IEEE Ultrason. Symp., pp. 1-4, 2004.

Michiel Postema e-mail: postema@ieee.org 